\title{
Individual Differences in True and False Memory Retrieval Are Related to White Matter Brain Microstructure
}

\author{
Lluís Fuentemilla, ${ }^{1 *}$ Estela Càmara, ${ }^{1,2 *}$ Thomas F. Münte, ${ }^{2,3}$ Ulrike M. Krämer, ${ }^{2}$ Toni Cunillera, ${ }^{1}$ Josep Marco-Pallarés, ${ }^{2}$ \\ Claus Tempelmann, ${ }^{4}$ and Antoni Rodriguez-Fornells ${ }^{1,5}$ \\ ${ }^{1}$ Departament de Ciències Fisiològiques II, University of Barcelona, Institut d'Investigació Biomèdica de Bellvitge, 08907 L'Hospitalet de Llobregat \\ (Barcelona), Spain, ${ }^{2}$ Department of Neuropsychology, ${ }^{3}$ Center for Behavioral Brain Sciences, and ${ }^{4}$ Department of Neurology, Otto von Guericke University, \\ 39120 Magdeburg, Germany, and 5 Institució Catalana de Recerca i Estudis Avançats, 08010 Barcelona, Spain
}

We sometimes vividly remember things that did not happen, a phenomenon with general relevance, not only in the courtroom. It is unclear to what extent individual differences in false memories are driven by anatomical differences in memory-relevant brain regions. Here we show in humans that microstructural properties of different white matter tracts as quantified using diffusion tensor imaging are strongly correlated with true and false memory retrieval. To investigate these hypotheses, we tested a large group of participants in a version of the Deese-Roediger-McDermott paradigm (recall and recognition) and subsequently obtained diffusion tensor images. A voxel-based whole-brain level linear regression analysis was performed to relate fractional anisotropy to indices of true and false memory recall and recognition. True memory was correlated to diffusion anisotropy in the inferior longitudinal fascicle, the major connective pathway of the medial temporal lobe, whereas a greater proneness to retrieve false items was related to the superior longitudinal fascicle connecting frontoparietal structures. Our results show that individual differences in white matter microstructure underlie true and false memory performance.

\section{Introduction}

Individual differences in the way people retrieve past events from memory are well documented (Blair et al., 2002; Watson et al., 2005). Because of the constructive nature of human memory, the recollective process may sometimes elicit misattributions that a novel event or experience has occurred previously, thus creating a false memory (FM) (Schacter et al., 1996). False memories (FM) can be easily elicited in the laboratory (Roediger and McDermott, 1995). For example, in the Deese-Roediger-McDermott (DRM) paradigm, lists of semantically related words are presented during encoding (e.g., seat-sofa-stool-table, etc.) with one prototypical exemplar of the category ("lure" word: chair) missing. Interestingly, the lure word is often produced in free recall or recognition tests. True memory (TM) recognition has been hypothesized to rely on accurate, context-rich and vivid retrieval of an event (i.e., recollection), whereas FM recognition appears to reflect the feeling of knowing something without specific contextual details and the semantic gist of the list (Brainerd and Reyna,

Received Nov. 1, 2008; revised May 18, 2009; accepted May 22, 2009.

This work was supported by the Volkswagen-Foundation, Ministerio de Educación y Ciencia, and Deutsche Forschungsgemeinschaft (Sonderforschungsbereich 779 TPA7). J.M.-P. is a fellow of the Humboldt Foundation. Bundesministerium für Bildung und Forschung is gratefully acknowledged. We thank D. Cucurell and A. Mestres for their help during data acquisition.

*L.F. and E.C. contributed equally to this work.

Correspondence should be addressed to Antoni Rodriguez-Fornells, Departament de Ciències Fisiològiques II, Campus de Bellvitge, University of Barcelona, Institut d'Investigació Biomèdica de Bellvitge, Feixa Llarga s/n, 08907 L'Hospitalet de Llobregat (Barcelona), Spain. E-mail: antoni.rodriguez@icrea.es.

L. Fuentemilla's present address: Institute of Cognitive Neuroscience, University College London, London WC1N 3AR, UK.

DOI:10.1523/JNEUROSCI.5270-08.2009

Copyright $\odot 2009$ Society for Neuroscience $\quad$ 0270-6474/09/298698-06\$15.00/0
2002). Recollection and familiarity- or gist-based retrieval are qualitatively different processes subserved by different neural structures (Sauvage et al., 2008), and recently it has been shown by functional neuroimaging that TM and FM retrieval in the DRM paradigm are mediated by different neural mechanisms (Kim and Cabeza, 2007). Specifically, highly confident TM recognitions are supported by the medial temporal lobe, a structure that has been related to recollection, whereas highly confident FM recognition engage frontoparietal regions, which are thought to mediate familiarity-based memory retrieval (Kim and Cabeza, 2007).

Importantly for the present investigation, this tendency to produce FM shows marked stable individual differences across time (Blair et al., 2002; Watson et al., 2005). Age-related differences across the lifespan in FM have also been associated with differences in brain activation (Dennis et al., 2008; Paz-Alonso et al., 2008). In children, age-related increases in TM are associated with changes in the medial temporal lobe (MTL), whereas increases in FM are related with activation changes in ventrolateral prefrontal cortex (Paz-Alonso et al., 2008). In older adults, TM recognition leads to weaker activity in the hippocampus compared with young controls, whereas FM is associated with increased activity in the left middle temporal gyrus, a region involved in semantic processing and semantic gist (Dennis et al., 2008).

In the present investigation, we asked whether individual differences in TM/FM retrieval may be related to differences in the organization of white matter connections [defined as per fractional anisotropy (FA) values derived from diffusion tensor magnetic resonance images (Le Bihan, 2003)]. To the extent to which 
the functioning of critical brain areas for FM and TM retrieval is dependent on the organization of their connecting fiber tracts, we predicted that (1) better TM retrieval should be positively correlated to FA in the inferior longitudinal fascicle (ILF), supporting the functions of medial temporal lobe structures, whereas (2) a greater susceptibility to FM retrieval should be related to FA in the superior longitudinal fascicle (SLF), which connects frontoparietal structures.

\section{Materials and Methods}

Participants. Forty-eight healthy, right-handed students [32 females; mean \pm SD age, $21.2 \pm 2.8$ years] from the University of Barcelona participated in this study after giving written informed consent. The study was approved by the University of Barcelona ethics committee.

Procedure and materials. The memory paradigm consisted of four lists of semantically related words presented via loudspeakers at the rate of one word every $2 \mathrm{~s}$. Each list comprised 14 semantically related Spanish words translated from the original DRM study (Roediger and McDermott, 1995) (supplemental data, available at www.jneurosci.org as supplemental material). After each list, participants were required to recall as many words as possible by writing them on an unmarked sheet of white paper ("recall phase"). Approximately $20 \mathrm{~min}$ after the recall phase, participants were required to judge whether a word had appeared in one of the four previous study lists (old vs new decision) from a list of 16 words ("recognition phase"). The recognition list comprised eight "studied words" (the first and eighth words of each studied lists), the four words semantically associated with each list ("lures"), and four words neither presented during the study phase or semantically related to any of the studied words ("new words"). To avoid possible biases when comparing lure words with other new words (Gallo and Roediger, 2002), the new words were translated from the set of lures for other lists in the original DRM study that were not studied in the present experiment.

In the case of "old" decisions, participants were instructed to make a "remember-know" judgment, which allows an evaluation of the subjective experience accompanying recognition judgments (Tulving, 1985). Participants were instructed to mark "remember" if they had a conscious and vivid recollection of the words from the study list (i.e., participants remembered what they were thinking about at the time the word was presented, the particular order in which the word was presented, or the physical characteristics associated with the presentation of the word). Participants were instructed to make a "know" judgment when they knew the word was presented but could not recollect its actual occurrence or any related details of its presentation. The experimenter ensured that all participants fully understood the distinction between the remember and know judgments before the recognition test. Percentage of TM and FM recall and recognition words were then calculated individually (supplemental data, available at www.jneurosci.org as supplemental material).

Magnetic resonance imaging-diffusion tensor imaging protocol. Diffusion tensor imaging (DTI) data were collected using a $3 \mathrm{~T}$ magnetic resonance imaging (MRI) scanner (Siemens Magnetom Trio) using an eight-channel phased array head coil with parallel imaging (GRAPPA) and an acceleration factor of 2 . Diffusion weighting was conducted using the standard twice refocused spin echo sequence. Images were measured using the following: 2-mm-thick slices; no gap; repetition time, $8200 \mathrm{~ms}$; echo time, $85 \mathrm{~ms} ; 128 \times 128$ acquisition matrix; field of view, $256 \times 256$ $\mathrm{mm} ; 64$ axial slices. To obtain diffusion tensors, diffusion was measured along 12 non-collinear directions, chosen according to the standard Siemens DTI acquisition scheme using a single $b$ value of $1000 \mathrm{~s} / \mathrm{mm}^{2}$. Two signal averages and three runs were acquired per slice and diffusion gradient direction. Each run was preceded by a non-diffusion-weighted volume for purposes of registration for motion correction.

Preprocessing of diffusion-weighted data. DTI data were motion corrected, and eddy current-induced distortions were removed before the estimation of the diffusion tensors. The first non-diffusion-weighted image of each block was realigned with the first image of the first series. Then, the determined transformation parameters were applied to the remaining diffusion-weighted images of the respective block. Subse- quently, all images were averaged across the three runs. To assess FA values, using the SPM2 diffusion toolbox (http://www.fil.ion.ucl.ac.uk/ $\mathrm{spm} /$ ), diffusion tensor elements were extracted from an overdetermined set of diffusion-weighted images. Diffusion tensors were diagonalized, and the eigenvectors and eigenvalues were obtained. Based on these eigenvalues, FA was calculated on a voxelwise basis. Normalization of the FA data was performed based on the FA images without Jacobian modulation of the signal intensities as reported previously (Càmara et al., 2007) (supplemental data, available at www.jneurosci.org as supplemental material).

Statistical analysis. The voxelwise analysis aimed at detecting the voxels in which FA values correlated with memory-related measures. FA values range from 0 to 1 , with larger values indicating that water diffusion occurs preferentially along one direction, as is the case for the structural organization of white matter tracts (Le Bihan, 2003). Previously normalized FA images were independently regressed on the proportion of true and false memory recall and recognition scores by applying a simple regression model in SPM2. This analysis was constrained to those voxels with FA values $>0.15$ in each single participant. This cutoff allowed to reliably isolate white matter from other brain tissues (Jones et al., 1999). Locations and significance levels from the correlation analysis were restricted to three different thresholds using the criterion of at least 60 contiguous voxels with $p<0.05, p<0.01$, and $p<0.005$. The use of this gradual threshold allows the visualization of the underlying white matter path. However, only regions significant at $p<0.005$ at voxel level (uncorrected) are reported in Table 1 and discussed in text. Whole-brain false discovery rate correction is also reported when significant $(p<$ 0.05 ). The maximum of suprathreshold regions were labeled using a white matter fiber DTI brain atlas (Wakana et al., 2004).

Region-of-interest (ROI) analysis was performed to confirm the voxel-based findings. Therefore, from the main peak correlations, FA values were extracted and correlated (Pearson's $r$ ) with memory-related measures. To ensure that the observed effects were not affected by outliers, values that deviated more than \pm 2.5 SDs from the mean ROI FA values or the behavioral scores were removed from the corresponding correlation. However, for all ROIs listed in Figure 2, highly similar results were obtained with and without outlier exclusion.

Finally, a direct comparison between the TM and FM correlation coefficients and the anisotropy coefficient was applied in the selected ROIs by using the Fisher's $z$ test for dependent correlations.

\section{Results}

\section{Memory performance}

A robust FM effect was obtained in the recall phase: lure words were falsely recalled in approximately half of the cases (FM recall) $(46 \pm 26 \%$ ), whereas studied words (TM recall) were recalled in $74 \pm 9 \%$ of the cases $\left(t_{(47)}=6.53 ; p<0.001\right)$. FM and TM recall measures were not correlated $(r=0.08)$, which is in line with the view that they are at least partially mediated by distinct retrieval processes (Brainerd and Reyna, 2002).

In the recognition phase, the majority of lure words was falsely recognized as studied words (FM recognition) (75 $\pm 27 \%)$. Performance was almost perfect for the recognition of the studied words (TM recognition, $94 \pm 9 \%$; comparison between FM and TM recognition, $\left.t_{(47)}=4.61 ; p<0.001\right)$. False old decisions for unrelated new words were very rare $(2 \pm 7 \%$ ) (FM recognition vs unrelated new-words, $\left.t_{(47)}=17.17 ; p<0.001\right)$. For recognized studied words (TM), a remember judgment was obtained in $78 \pm$ $20 \%$ of the cases, indicating a conscious recollection and vivid memory experience elicited for these studied words. There were significantly less remember judgments for FM (58 $\pm 36 \% ; t_{(47)}=$ 4.43; $p<0.001)$. However, TM and FM remember scores were significantly correlated $(r=0.55 ; p<0.01)$. This could be explained by a tendency to use a semantic generation strategy to recollect TM, which should also lead to increased FM (Winograd et al., 1998). 
Table 1. White matter changes associated with TM and FM recall and recognition

\begin{tabular}{|c|c|c|c|c|c|c|c|c|}
\hline \multirow[b]{2}{*}{ Cluster } & \multirow[b]{2}{*}{ Side } & \multirow[b]{2}{*}{ Cluster size $\left(\mathrm{mm}^{3}\right)$} & \multirow[b]{2}{*}{$r$} & \multirow[b]{2}{*}{ Slope (FA/s) } & \multirow[b]{2}{*}{$t$ value } & \multicolumn{3}{|c|}{ Peak coordinate } \\
\hline & & & & & & $x$ & $y$ & $z$ \\
\hline \multicolumn{9}{|l|}{ True memories } \\
\hline \multicolumn{9}{|l|}{ Recall } \\
\hline \multirow[t]{2}{*}{ ILF } & $\mathrm{R}$ & 1486 & 0.39 & 0.08 & 3.75 & 46 & -25 & -13 \\
\hline & $\mathrm{L}$ & 631 & 0.39 & 0.11 & 4.22 & -44 & -25 & 0 \\
\hline IFO/ILF & $\mathrm{L}$ & 983 & 0.44 & 0.12 & 3.98 & -33 & -76 & -4 \\
\hline \multicolumn{9}{|l|}{ Recognition } \\
\hline \multirow[t]{2}{*}{ ILF } & $\mathrm{R}^{*}$ & 5953 & 0.46 & 0.07 & 5.43 & 40 & -46 & -6 \\
\hline & $L^{* *}$ & 3818 & 0.35 & 0.06 & 4.86 & -37 & -52 & -3 \\
\hline \multicolumn{9}{|l|}{ False memories } \\
\hline \multicolumn{9}{|l|}{ Recall } \\
\hline SLF anterior & $\mathrm{R}$ & 829 & 0.43 & 0.07 & 3.91 & 9 & 21 & 15 \\
\hline SLF medial & $\mathrm{R}$ & 918 & 0.42 & 0.04 & 3.50 & 17 & -1 & 34 \\
\hline SLF posterior & $\mathrm{L}$ & 1500 & 0.45 & 0.05 & 3.65 & -18 & -34 & 33 \\
\hline IFO/ILF & $\mathrm{R}$ & 1569 & 0.33 & 0.03 & 3.93 & 45 & -54 & -8 \\
\hline \multicolumn{9}{|l|}{ Recognition } \\
\hline SLF medial & $\mathrm{R}$ & 1052 & 0.41 & 0.05 & 3.16 & 21 & 15 & 22 \\
\hline
\end{tabular}

All peak values reported were significant at $p<0.005$ (uncorrected); 60 voxels spatial extent. Correlation coefficients $(r)$ were calculated with the voxels comprising each cluster. For slope measure $\mathrm{FA} / \mathrm{s}$, $s$ refers to the regressed memory scores. Peak coordinates of each cluster are reported in Montreal Neurological Institute coordinates. IF0, Inferior fronto-occipital fascicle; R, right; $L$, left. * indicates regions that showed $p$ values false discovery rate corrected at the whole-brain level $(p<0.05)$ and at the cluster level $(p<0.001) .{ }^{* *}$ indicates regions that showed $p$ values false discovery rate corrected at the whole-brain level $(p<0.05)$ and at the cluster level $(p<0.01)$.

\section{DTI and memory performance}

Distinct patterns of correlation were obtained between FA and FM as well as FA and TM for both recall and recognition tests (Fig. 1 a,c, Table 1). Significant positive correlations between recall (and recognition, respectively) TM and FA were found bilaterally in regions coinciding with the ILF near MTL regions, including the hippocampus and parahippocampal structures that are known to play a crucial role for recollection in animals (Sauvage et al., 2008) and humans (Kim and Cabeza, 2007; Dennis et al., 2008). This fascicle extends from the ventral and lateral temporal regions to the posterior parahippocampal gyrus and has been associated to memory processes, as well as object and face processing (Schmahmann et al., 2007).

Recall (and recognition, respectively) FM, conversely, was correlated with FA in several parts of the SLF (Fig. 1 $a, c$, Table 1). The SLF is the principal connection between frontal and postrolandic parietal and temporal (superior and medial) association regions (Catani et al., 2005; Makris et al., 2005), lateral to the cingulum bundle. It thus connects several gray matter areas implicated in familiarity-based memory retrieval processes (Yonelinas et al., 2005). Whereas recall FM yielded positive correlations for several parts of the SLF as well as for the ILF, correlations for recognition FM were restricted to just the SLF.

An additional regression analysis was performed between memory scores and mean FA values extracted from the clusters found in the whole-brain analysis at $p<0.005$ (Table 1). Each cluster associated with either FM or TM recall (and recognition, respectively) scores showed significant positive correlation (all $p<0.01$ ), whereas no significant correlation resulted when clusters associated with TM in which correlated with FM scores and vice versa (all $p>0.1$ ) (Fig. 2). Representative regression analyses for TM and FM scores are shown in Figure 1, $b$ and $d$. The Fisher's $z$-test comparison between the TM and FM correlation coefficients confirmed significant differences in all of the clusters defined in the whole-brain analysis (all $p<0.05$, one-tailed) except for the SLF cluster for the recognition phase [21 15 22].

\section{Discussion}

In the present study, we observed that interindividual differences in TM and FM were associated with differences in the microstructural properties of two dissociable fiber tracts. Greater FA in the ILF, connecting MTL structures, is related to higher TM scores. This result dovetails nicely with functional imaging results of greater MTL activity for TM (Kim and Cabeza, 2007; Dennis et al., 2008). Greater FA in the SLF, connecting frontal and parietal structures, is related to increased susceptibility to FM, which again coincides with functional MRI findings of greater activation in frontoparietal regions for FM decisions (Kim and Cabeza, 2007). In line with the idea that anatomical circuitry in the brain might constrain the nature of information processing locally and across brain regions (Behrens and Johansen-Berg, 2005), we have observed that local white matter microstructure differences surrounding discrete functional regions show a strong relationship with different memory performance measures.

Using DTI, it has been shown recently that FA predicts behavioral patterns or functional brain activations in other domains, such as decision making (Boorman et al., 2007) or language (Gold et al., 2007). With regard to the current results, innate differences in white matter structure might explain differences in FM and TM retrieval processes. Alternatively, variations in experience or cognitive style might induce neural plasticity of white matter tracts. FA values are thought to reflect axonal microstructure in vivo, for example, axon size and the extent of myelinization (Basser and Jones, 2002), which affect the quality of axonal transmission (Waxman and Bennett, 1972) and are susceptible to experience-dependent changes (Demerens et al., 1996; Fields, 2008). Changes in FA have been shown in professional pianists, possibly reflecting the neural reorganization that occurs over the course of long-term musical training (Bengtsson et al., 2005). This increase in white matter microstructure was dependent on the number of hours each subject had practiced the instrument, which suggests the involvement of myelin plasticity in the acquisition and learning of specific skills.

The strong correlation reported between the ILF and TM implies the involvement of white matter pathways surrounding the MTL region in recollective memory processes. Indeed, the MTL has been demonstrated to be critical for recollection in both animal (Fortin et al., 2004) and human (Eichenbaum et al., 2007) studies. Lesions in MTL regions impaired recollection but not familiarity (Yonelinas et al., 2002), and neuroimaging data revealed that this pattern of results has been consistent across 


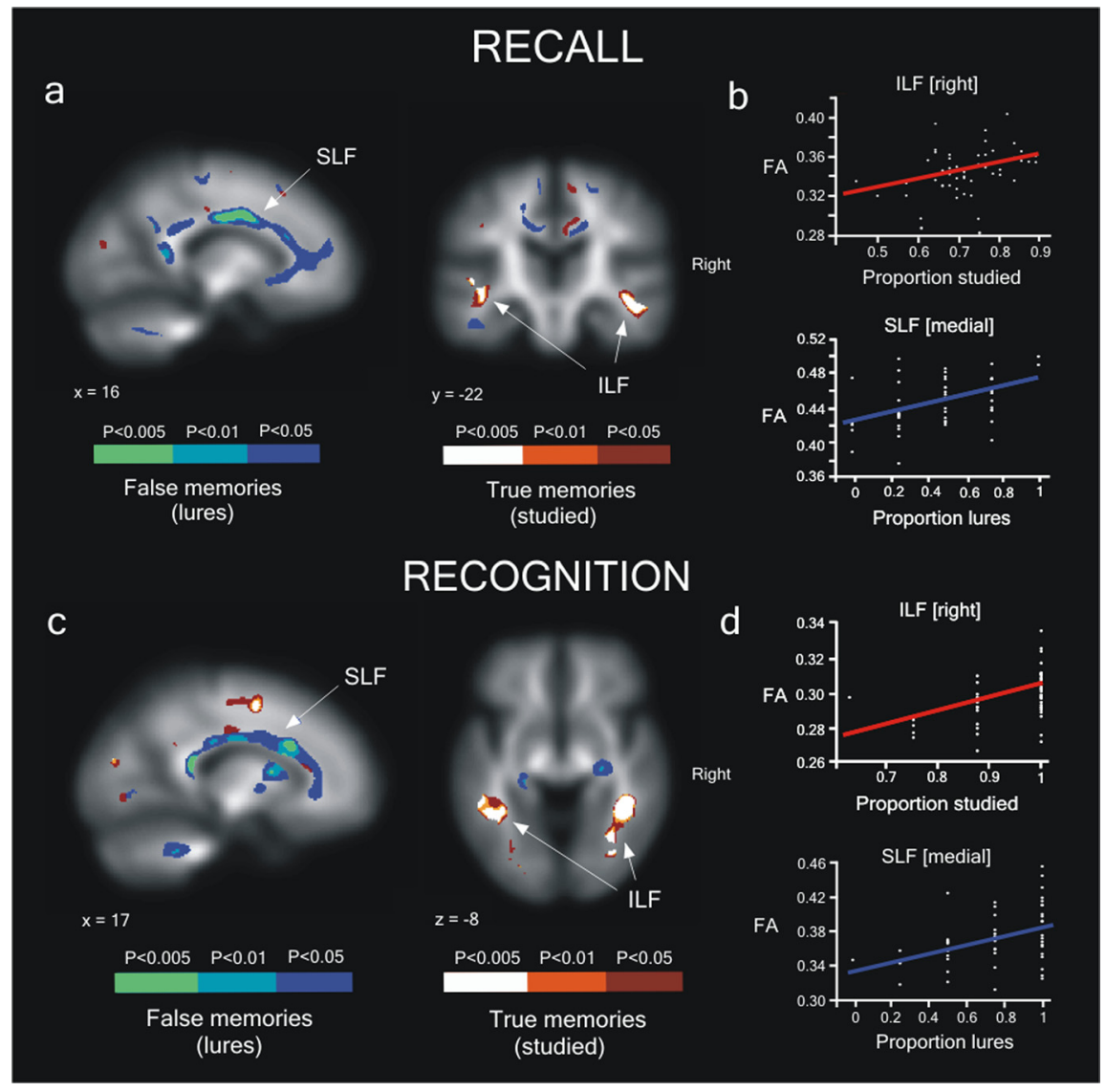

Figure 1. Individual differences in TM and FM free recall/recognition scores are correlated to FA of major white matter tracts. $\boldsymbol{a}$, Recall: Significant white matter-correlated clusters rendered on the FA mean image ( $p<0.005 ; n=60$ voxels spatial extent). The same results are shown with a more liberal statistical threshold to visualize the white matter pathways $(p<0.01 ; p<0.05$ $n=60)$. $\boldsymbol{b}$, Relationship between the mean FA value for each participant in two selected ROls and TM recall (peak Montreal Neurological Institute coordinates: right ILF, $x=46, y=-25, z=-13$ ) and FM recall (right SLF, $x=17, y=-1, z=34$ ). $c$, Significant white matter clusters that correlated with the proportion of TM and FM recognition. $d$, Relationship between the mean FA value for each participant in two selected ROIs and TM recognition (right ILF, $x=40, y=-46, z=-6$ ] and FM recognition (right SFL, $x=21, y=15, z=22$ ). Red and blue lines represent regression lines for TM and FM recognition, respectively.
Cabeza, 2007), the current results appear compatible with the fuzzy trace theory.

In contrast, the activation-monitoring framework (Balota et al., 1999) stresses the need to differentiate between highly activated but nonpresented critical words and studied words to avoid FM. By this account, FMs are attributable to a failure in monitoring processes differentiating the activation of lure words in associative networks from the actual presentation of words at encoding (Balota et al., 1999). The dorsolateral prefrontal cortex is more activated during false than true recognition, which has been interpreted as reflecting monitoring processes induced by the strong sense of familiarity associated with FM (Schacter et al., 1996). The current finding that greater FA in the SLF is associated with more FM is difficult to reconcile with an activation-monitoring account, however, because greater FA values have been interpreted as reflecting the extent of myelination and axonal density within axonal bundles, which in turn suggests better functionality of the target brain areas. Better functionality of retrieval monitoring systems should thus lead to decreased FM, which is the opposite of what we observed. Because the SLF projects extensively to different parts of the prefrontal cortex that are thought to play a differential role in monitoring and semantic retrieval, increased processing efficiency in areas such as the ventrolateral prefrontal cortex associated with semantic elaboration and retrieval control might raise the amount of false memories. This would also fit with recent functional MRI different measurement techniques and different stimulus materials when remember-know responses were compared (Yonelinas et al., 2005) but also when graded MTL activity has been shown as a function of confidence judgments (Yonelinas et al., 2005). In agreement with that, Kim and Cabeza (2007) interpreted MTL activity during high-confidence true recognition as reflecting mainly recollection in a DRM paradigm. Our ILF results strongly support this view because TMs were also associated with a higher percentage of remember responses. Furthermore, they also emphasize the central role of ILF in explaining recollection-based individual differences.

In relation to FM, two major theoretical accounts have been advanced to explain this phenomenon: the fuzzy trace theory and the action-monitoring account. According to the former (Brainerd and Reyna, 2002), stimuli are encoded into two qualitatively different types of memory traces: a "verbatim" trace, which contains item-specific details (perceptual attributes, position in the list, etc.), and a "gist" trace, which represents episodic conceptual information (e.g., thematic similarity or conceptual overlap between the words presented in the list). The verbatim trace gives rise to TM, whereas gist-based information may lead to FM. To the extent to which verbatim traces are supported by MTL structures and communicated via the ILF and gist-based information is mediated by frontoparietal structures and the SLF (Kim and findings of enhanced ventrolateral prefrontal cortex activity during false memory recognition (Paz-Alonso et al., 2008). Thus, the present results are not specific enough to discard the activationmonitoring account.

Furthermore, several studies have already observed greater activation in several brain regions (including the prefrontal cortex, medial and inferior parietal cortex) for subsequently forgotten relative to remembered (Wagner and Davachi, 2001; Daselaar et al., 2004; Shrager et al., 2008). This activation increase has been proposed to be related to an increase in the default-mode network activation (Shrager et al., 2008), associated with mindwandering or lapses of attention (Weissman et al., 2006; Mason et al., 2007). Shrager et al. (2008) have proposed that activation of this network might explain the negative correlation observed between recognition memory strength and the activation observed in inferior parietal cortex, right anterior and posterior prefrontal cortex, and medial parietal cortex. Because of the increased activity of the default-mode network during learning, recollectionbased recognition decreases (mediated by MTL regions) and participants might be forced to depend on familiarity-based processes. Our positive association between FA in the SLF and FM recall and recognition scores could then reflect the participants' susceptibility to mind-wandering and inattention during the learning phase. 


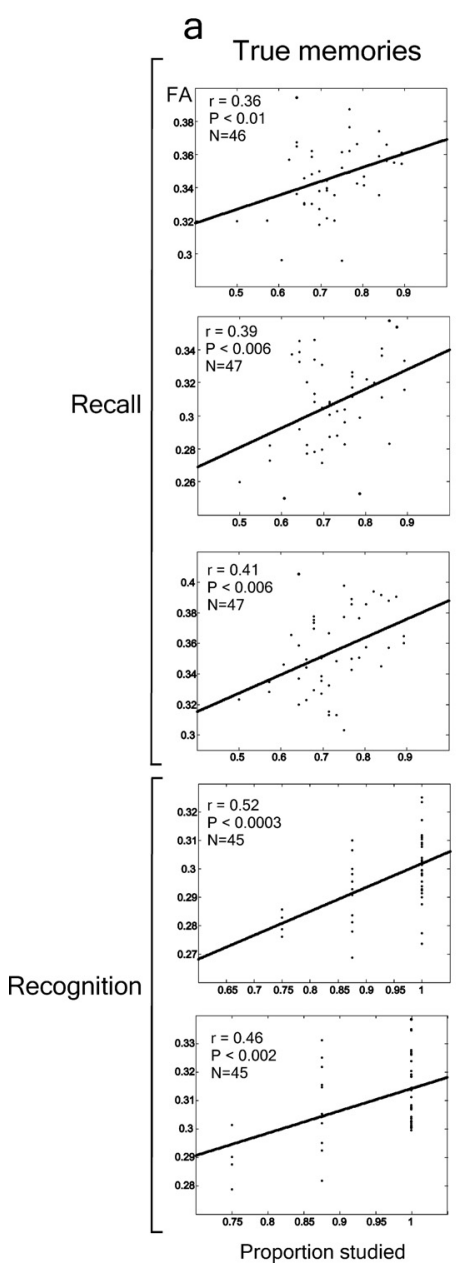

False memories
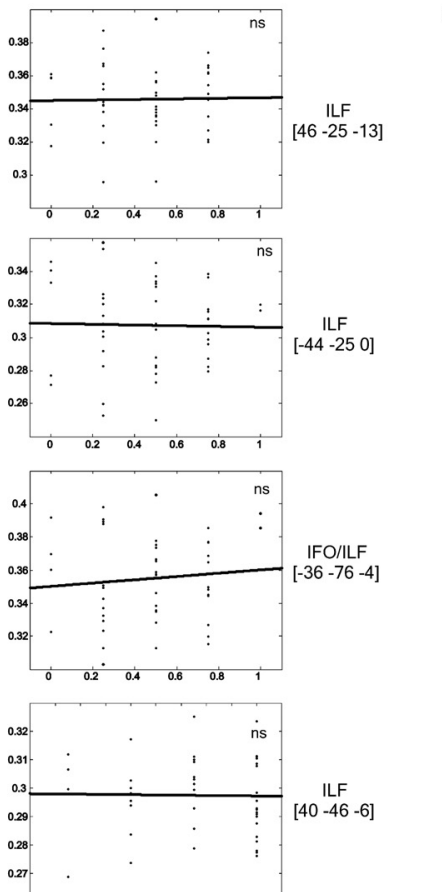

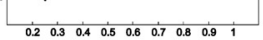

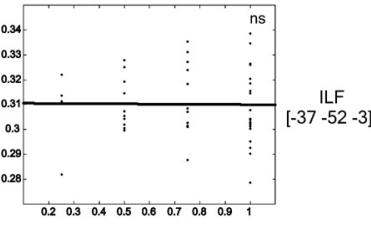

Proportion lures

b

True memories
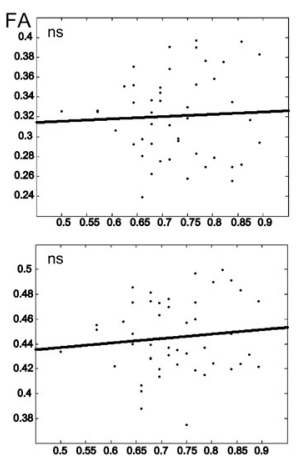

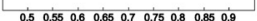
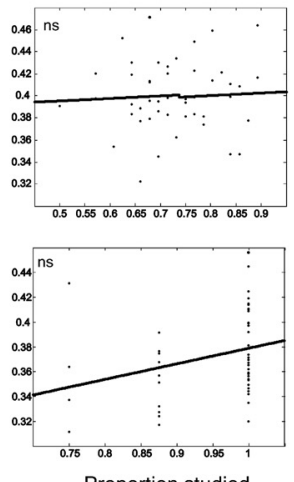

Proportion studied
False memories
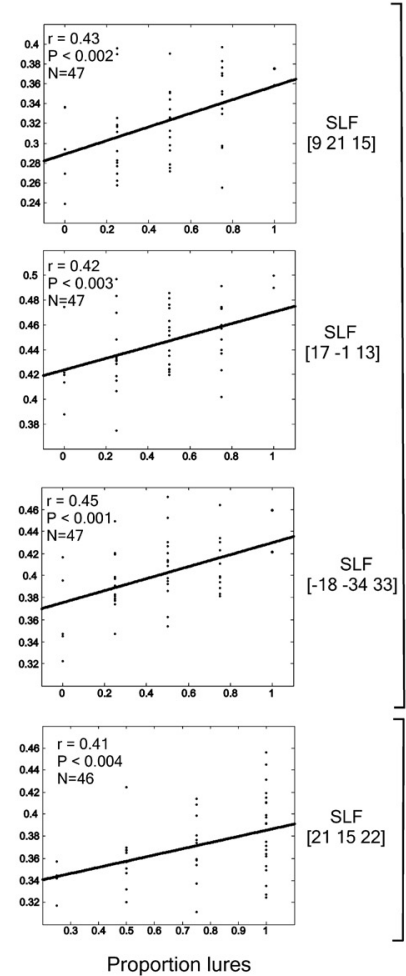

Recall

Recognition

Figure 2. Correlations between FA and TM or FM recall and recognition scores for the peak locations of the white matter regions described in Table 1. Whereas most of the peak locations corresponding to the ILF correlated with TM recall and recognition scores, no correlation was observed with FM scores $(\boldsymbol{a})$. The reverse pattern was observed for SLF regions $(\boldsymbol{b})$. Nonsignificant correlations are marked by "ns".

A question of potential clinical relevance is whether long-term training might induce white matter changes associated with strategic use of memory retrieval processes and thus would lead to reduced FM. With regard to gray matter, several studies have recently shown learning-induced changes using structural MRI (Maguire et al., 2000; Draganski et al., 2004). While we await results of such studies, the present experiment already demonstrates the relevance of studying network interconnectivity and white matter structural differences to understand individual variations in cognitive functions in general and memory functions in particular. The DTI technique is suitable for the assessment of such microstructural changes (Beaulieu, 2002) and plasticityrelated processes (Le Bihan, 2003; Tovar-Moll et al., 2007) induced by long-term learning experiences.

\section{References}

Balota DA, Cortese MJ, Duchek JM, Adams D, Roediger HL, McDermott KB, Yerys BE (1999) Veridical and false memories in healthy older adults and in dementia of the Alzheimer's type. Cogn Neuropsychol 16:361-384.

Basser PJ, Jones DK (2002) Diffusion-tensor MRI: theory, experimental design and data analysis-a technical review. NMR Biomed 15:456-467.

Beaulieu C (2002) The basis of anisotropic water diffusion in the nervous system: a technical review. NMR Biomed 15:435-455.

Behrens TE, Johansen-Berg H (2005) Relating connectional architecture to grey matter function using diffusion imaging. Philos Trans R Soc Lond B Biol Sci 360:903-911.
Bengtsson SL, Nagy Z, Skare S, Forsman L, Forssberg H, Ullén F (2005) Extensive piano practicing has regionally specific effects on white matter development. Nat Neurosci 8:1148-1150.

Blair IV, Lenton AP, Hastie R (2002) The reliability of the DRM paradigm as a measure of individual differences in false memories. Psychon Bull Rev 9:590-596.

Boorman ED, O'Shea J, Sebastian C, Rushworth MF, Johansen-Berg H (2007) Individual differences in white-matter microstructure reflect variation in functional connectivity during choice. Curr Biol 17:1426-1431.

Brainerd CJ, Reyna VF (2002) Fuzzy-trace theory and false memory. Curr Dir Psychol Sci 11:164-169.

Càmara E, Bodammer N, Rodríguez-Fornells A, Tempelmann C (2007) Age-related water diffusion changes in human brain: a voxel-based approach. Neuroimage 34:1588-1599.

Catani M, Jones DK, ffytche DH (2005) Perisylvian language networks of the human brain. Ann Neurol 57:8-16.

Daselaar SM, Prince SE, Cabeza R (2004) When less means more: deactivations during encoding that predict subsequent memory. Neuroimage 23:921-927.

Demerens C, Stankoff B, Logak M, Anglade P, Allinquant B, Couraud F, Zalc B, Lubetzki C (1996) Induction of myelination in the central nervous system by electrical activity. Proc Natl Acad Sci U S A 93:9887-9892.

Dennis NA, Kim H, Cabeza R (2008) Age-related differences in brain activity during true and false memory retrieval. J Cogn Neurosci 20:1390-1402.

Draganski B, Gaser C, Busch V, Schuierer G, Bogdahn U, May A (2004) 
Neuroplasticity: changes in grey matter induced by training. Nature 427:311-312.

Eichenbaum H, Yonelinas AP, Ranganath C (2007) The medial temporal lobe and recognition memory. Annu Rev Neurosci 30:123-152.

Fields RD (2008) White matter in learning, cognition and psychiatric disorders. Trends Neurosci 31:361-370.

Fortin NJ, Wright SP, Eichenbaum H (2004) Recollection-like memory retrieval in rats is dependent on the hippocampus. Nature 431:188-191.

Gallo DA, Roediger HL (2002) Variability among word lists in eliciting memory illusions: evidence for associative activation and monitoring. J Mem Lang 47:469-497.

Gold BT, Powell DK, Xuan L, Jiang Y, Hardy PA (2007) Speed of lexical decision correlates with diffusion anisotropy in left parietal and frontal white matter: evidence from diffusion tensor imaging. Neuropsychologia 45:2439-2446.

Jones DK, Lythgoe D, Horsfield MA, Simmons A, Williams SC, Markus HS (1999) Characterization of white matter damage in ischemic leukoaraiosis with diffusion tensor MRI. Stroke 30:393-397.

Kim H, Cabeza R (2007) Trusting our memories: dissociating the neural correlates of confidence in veridical versus illusory memories. J Neurosci 27:12190-12197.

Le Bihan D (2003) Looking into the functional architecture of the brain with diffusion MRI. Nat Rev Neurosci 4:469-480.

Maguire EA, Gadian DG, Johnsrude IS, Good CD, Ashburner J, Frackowiak RS, Frith CD (2000) Navigation-related structural change in the hippocampi of taxi drivers. Proc Natl Acad Sci U S A 97:4398-4403.

Makris N, Kennedy DN, McInerney S, Sorensen AG, Wang R, Caviness VS Jr, Pandya DN (2005) Segmentation of subcomponents within the superior longitudinal fascicle in humans: a quantitative, in vivo, DT-MRI study. Cereb Cortex 15:854-869.

Mason MF, Norton MI, Van Horn JD, Wegner DM, Grafton ST, Macrae CN (2007) Wandering minds: the default network and stimulusindependent thought. Science 315:393-395.

Paz-Alonso PM, Ghetti S, Donohue SE, Goodman GS, Bunge SA (2008) Neurodevelopmental correlates of true and false recognition. Cereb Cortex 18:2208-2216.

Roediger HL, McDermott KB (1995) Creating false memories: remembering words not presented in lists. J Exp Psychol Learn Mem Cogn 21:803-814.
Sauvage MM, Fortin NJ, Owens CB, Yonelinas AP, Eichenbaum H (2008) Recognition memory: opposite effects of hippocampal damage on recollection and familiarity. Nat Neurosci 11:16-18.

Schacter DL, Reiman E, Curran T, Yun LS, Bandy D, McDermott KB, Roediger HL 3rd (1996) Neuroanatomical correlates of veridical and illusory recognition memory: evidence from positron emission tomography. Neuron 17:267-274.

Schmahmann JD, Pandya DN, Wang R, Dai G, D'Arceuil HE, de Crespigny AJ, Wedeen VJ (2007) Association fibre pathways of the brain: parallel observations from diffusion spectrum imaging and autoradiography. Brain 130:630-653.

Shrager Y, Kirwan CB, Squire LR (2008) Activity in both hippocampus and perirhinal cortex predicts the memory strength of subsequently remembered information. Neuron 59:547-553.

Tovar-Moll F, Moll J, de Oliveira-Souza R, Bramati I, Andreiuolo PA, Lent R (2007) Neuroplasticity in human callosal dysgenesis: a diffusion tensor imaging study. Cereb Cortex 17:531-541.

Tulving E (1985) Memory and consciousness. Can Psychol 26:1-12.

Wagner AD, Davachi L (2001) Cognitive neuroscience: forgetting of things past. Curr Biol 11:R964-R967.

Wakana S, Jiang H, Nagae-Poetscher LM, van Zijl PC, Mori S (2004) Fiber tract-based atlas of human white matter anatomy. Radiology 230:77-87.

Watson JM, Bunting MF, Poole BJ, Conway AR (2005) Individual differences in susceptibility to false memory in the Deese-RoedigerMcDermott paradigm. J Exp Psychol Learn Mem Cogn 31:76-85.

Waxman SG, Bennett MV (1972) Relative conduction velocities of small myelinated and non-myelinated fibres in the central nervous system. Nat New Biol 238:217-219.

Weissman DH, Roberts KC, Visscher KM, Woldorff MG (2006) The neural bases of momentary lapses in attention. Nat Neurosci 9:971-978.

Winograd E, Peluso JP, Glover TA (1998) Individual differences susceptibility to memory illusions. Appl Cogn Psychol 12:S5-S27.

Yonelinas AP, Kroll NE, Quamme JR, Lazzara MM, Sauvé MJ, Widaman KF, Knight RT (2002) Effects of extensive temporal lobe damage or mild hypoxia on recollection and familiarity. Nat Neurosci 5:1236-1241.

Yonelinas AP, Otten LJ, Shaw KN, Rugg MD (2005) Separating the brain regions involved in recollection and familiarity in recognition memory. J Neurosci 25:3002-3008. 